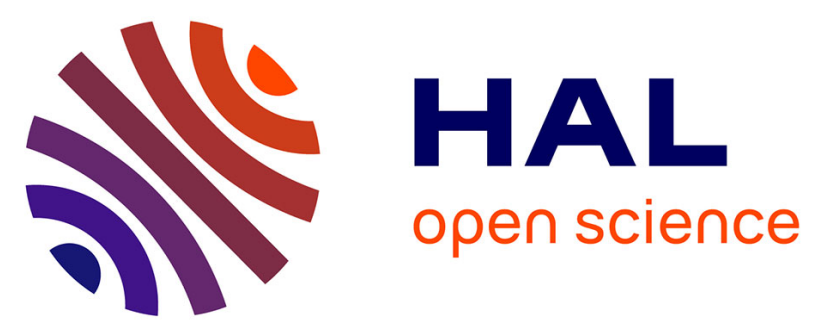

\title{
Improved 3D electromagnetic analytical model for planar induction heater with consideration of transverse edge effects
}

\author{
Mohammed Messadi, Larbi Hadjout, Youcef Ouazir, Hakim Bensaidane, \\ Noureddine Takorabet, Thierry Lubin, Smail Mezani
}

\section{To cite this version:}

Mohammed Messadi, Larbi Hadjout, Youcef Ouazir, Hakim Bensaidane, Noureddine Takorabet, et al.. Improved 3D electromagnetic analytical model for planar induction heater with consideration of transverse edge effects. COMPEL: The International Journal for Computation and Mathematics in Electrical and Electronic Engineering, 2020, 39 (5), pp.1169-1184. 10.1108/COMPEL-01-2020-0034 . hal-02880406

\section{HAL Id: hal-02880406 https://hal.science/hal-02880406}

Submitted on 25 Jun 2020

HAL is a multi-disciplinary open access archive for the deposit and dissemination of scientific research documents, whether they are published or not. The documents may come from teaching and research institutions in France or abroad, or from public or private research centers.
L'archive ouverte pluridisciplinaire $\mathbf{H A L}$, est destinée au dépôt et à la diffusion de documents scientifiques de niveau recherche, publiés ou non, émanant des établissements d'enseignement et de recherche français ou étrangers, des laboratoires publics ou privés. 


\title{
Improved 3D Electromagnetic Analytical Model for Planar Induction Heater with Consideration of Transverse Edge Effects

\author{
Mohammed Messadi, Larbi Hadjout, Youcef Ouazir, Hakim Bensaidane
}

LSEI-Université des Sciences et Technologie Houari Boumediene, BP N³2, 16111, Alger, Algérie

Noureddine Takorabet, Thierry Lubin, Smail Mezani

GREEN-Université de Lorraine, Faculté des Sciences et Technologies, BP 239, 54506, Vandœuvre-lès-Nancy, France

\begin{abstract}
Purpose - This paper proposes a new 3D electromagnetic model to compute translational motion eddy current in the conducting plate of a novel linear permanent magnet (PM) induction heater. The movement of the plate in a dc magnetic field created by a PM inductor generates induced currents that are at the origin of a heating power by Joule effect. These topologies have a strong magnetic end effects. The analytical model developed in this work takes into account the finite length extremity effects of the conducting plate and the reaction field due to induced currents.
\end{abstract}

Design/Methodology/Approach - The developed model is based on the combination of the sub-domain's method and the image's theory. Firstly, the magnetic field expressions due to the PMs is obtained by solving the three dimensional Maxwell equations by the method of separation of variables, using a magnetic scalar potential formulation and a magnetic field strength formulation. Then, the motional eddy currents are computed using the Ampere law and the finite length extremity effects of the conducting plate are taken into account using the image's method. To analyze the accuracy of the proposed model, the obtained results are compared to those obtained from 3D finite element model (3D FEM) and from experimental tests performed on a prototype.

Finding - The results show that the developed analytical model is very accurate, even for geometries where the edge effects are very strong. It allows directly taking into account the finite length extremity effects (the transverse edge effects) of the conducting plate and the reaction field due to induced currents without the need of any correction factor.

The proposed model presents also an important reduction in computation time compared to 3D finite element simulation, allowing fast analysis of linear permanent magnet (PM) induction heater.

Practical implications - The proposed electromagnetic analytical model can be used as a quick and accurate design tool for translational motion PM induction heater devices.

Originality/Value - A new 3D analytical electromagnetic model, to find the induced power in the conducting plate of a novel translational motion induction heater has been developed. The studied heating device has a finite length and a finite width which create edge effects which are not easily considered in calculation. The novelty of the presented method is the accurate 3D analytical model which allows finding the real power heating and real distribution of the induced currents in the conducting plate without the need to use correction factor. The proposed model also takes into account the reaction field due to induced currents.

In addition the developed model improves an important reduction in the computation time compared with 3D FEM simulation.

Keywords - eddy currents, induction heating, permanent magnets, three-dimensional, extremity effects, method of images, linear motion. 
Induction heating is widely used in many industrial applications such as heat treatment and forming processes (V. Rudnev \& al., 2003). These devices are applications of Lenz's Law and Joule Effect. The conducting object can be exposed to a variable magnetic field produced by AC winding (V. Rudnev \& al., 2003; Lubin \& al., 2009), or moved in a static magnetic field (Messadi \& al., 2016; Fabbri \& al., 2005; Mach \& al., 2014).

To improve the performance of these systems, new induction heating concepts have recently been proposed (Mach \& al., 2012; Mach \& al., 2014; Karban \& al., 2011, Boubezari \& al., 2014), using a permanent magnet (PM) inductor. In these topologies, the PM inductor produces a static magnetic field and the work piece to be heated is subjected to rotation or to linear movement.

The geometry of linear induction heating device is planar with finite dimensions. It has strong magnetic edge effects that need to be considered in the modeling (Messadi \& al., 2016; Bensaidane \& al., 2015; Paul \& al., 2014; Zhang \& al., 2014). Such devices can be modeled by 3D Finite Element Method (FEM) (Fabbri \& al., 2009; Selçuk \& al., 2008; Bíró \& al., 2011; Karban $\&$ al., 2011). However, this method has some drawbacks in terms of CPU time.

Many studies have been proposed to compute induction heating systems with rotating billets using 2D analytical models (Boubezari \& al., 2014, Lubin \& al., 2009; Fabbri \& al., 2009). These models are not suitable to take into account the edge effects and the power heating is not correctly evaluated. In (Bensaidane \& al., 2015), the authors developed a 2D analytical model where the end effects are taken into account using a correction factor.

This paper presents a new 3D electromagnetic analytical model to compute eddy currents and heating power in the conducting plate of a novel planar induction heater (Bensaidane \& al., 2015). The sub-domain's method and the image's theory are combined to compute the eddy currents with consideration of finite length effects and the reaction field. The boundary's conditions on the lateral faces of the conducting plate are taken into account thanks to the image's theory by exploiting the fictitious poles imposed by the fictitious anti-periodic condition. To analyze the accuracy of the proposed model, the obtained results are validated through those obtained from 3D finite element model and from experimental tests performed on a prototype.

The studied induction heating device is shown in Fig.1. It is composed of two permanent magnets (PM) inductors with quasi Halbach magnetization arrangement. A conducting plate subjected to a linear oscillatory motion is placed between these two inductors (Messadi \& al., 2016). The geometric parameters of the studied device are given in the Table. I.

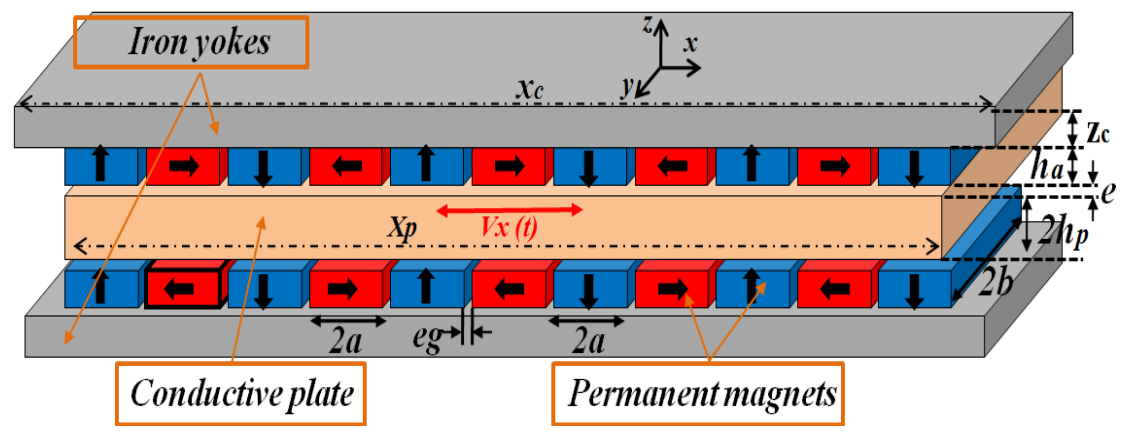

Fig. 1. Geometry of the studied device. 


\section{Computation method}

Solving the Maxwell equations in the 3-D Cartesian coordinates to calculate the eddy currents and heating power in the conducting plate are done in two steps:

- Firstly, we compute the magnetic flux density and the eddy current density for an infinite width conducting plate.

- Secondly, the image's theory is used to introduce the finite boundaries of the conducting plate in eddy currents components

TABLE I. GEOMETRIC PARAMETERS OF THE HEATER

\begin{tabular}{lll}
\hline Symbol & Quantity & Value \\
\hline $\mathbf{2 h}_{\mathbf{p}}$ & Conducting plate thickness & $15 \mathrm{~mm}$ \\
$\mathbf{X}_{\mathbf{p}}$ & Conducting plate length & $240 \mathrm{~mm}$ \\
$\mathbf{Y}_{\mathbf{p}}$ & Conducting plate width & $50 \mathrm{~mm}$ \\
$\mathbf{e}$ & air gap & $3 \mathrm{~mm}$ \\
$\mathbf{e g}$ & Distance between two magnets & $2 \mathrm{~mm}$ \\
$\mathbf{h a}$ & Permanent magnet thickness & $10 \mathrm{~mm}$ \\
$\mathbf{2 a}$ & Longitudinal and Transversal PM width & $20 \mathrm{~mm}$ \\
$\mathbf{2 b}$ & Permanent magnet length & $50 \mathrm{~mm}$ \\
$\mathbf{z}_{\mathbf{c}}$ & Iron yoke thickness & $20 \mathrm{~mm}$ \\
$\mathbf{x}_{\mathbf{c}}$ & Iron yoke length & $400 \mathrm{~mm}$ \\
$\mathbf{y}_{\mathbf{c}}$ & Iron yoke width & $60 \mathrm{~mm}$ \\
$\mathbf{V}_{\mathbf{x}}(\mathbf{t})$ & Oscillatory linear velocity & Variable \\
$\mathbf{V}_{\mathbf{m a x}}$ & Velocity peak value & Variable \\
$\boldsymbol{B r}$ & Residual induction of permanent magnets & $1.21 \mathrm{~T}$ \\
$\boldsymbol{\sigma}$ & Aluminum conductivity at $20^{\circ} \mathrm{C}$ & $33.1610^{6} \mathrm{Sm}^{-1}$ \\
\hline
\end{tabular}

\subsection{Magnetic flux density}

We assume that the iron-yokes have an infinite permeability. In these conditions and by considering the geometric symmetry of the device, the whole domain of the field problem is divided into three regions: Magnets region (I), the air-gap (II) and the conducting plate (III), (Fig. 2).

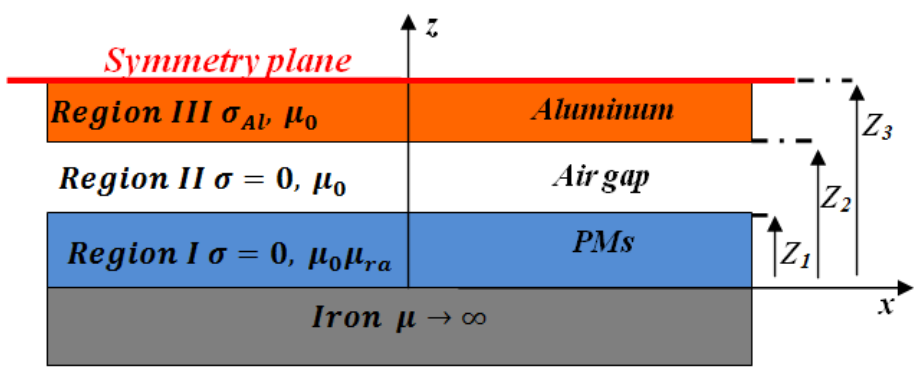

Fig. 2. Geometrical model.

To solve the 3-D boundary value problem, the boundary conditions of our system in the $\mathrm{x}$ - and $\mathrm{y}$-directions must be defined. Due to the alternate polarity of the PMs the boundary condition in the $\mathrm{x}$-direction is naturally anti-periodic. The same anti-periodic condition is applied at the boundaries on the y-direction, while respecting the ly >>b (Fig.3), to avoid any magnetic coupling between the fictitious poles along the y axis. This boundary condition allows to cancel the magnetic flux density at $y= \pm$ ly (Fig.3) (Lubin \& al., 2015). 


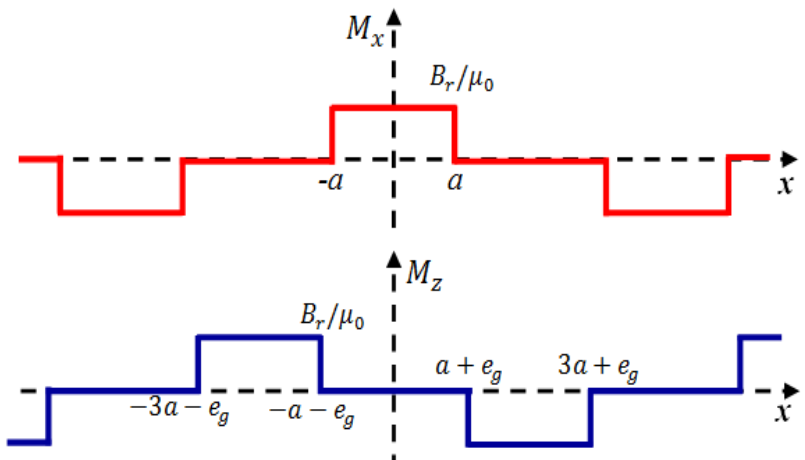

(a)

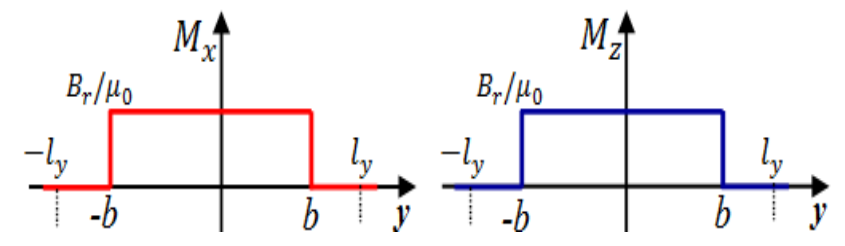

(b)

Fig. 3. Magnetization distribution along the $x$ - and $y$-direction.

To solve this problem, a magnetic scalar potential formulation is used in both magnets and air gap regions and a magnetic field strength formulation in the conducting plate.

Taking into account the presence of permanent magnets with $(M x)$ and $(M z)$ magnetization, the magnetic scalar potentials $\phi_{\mathrm{I}}$ in the magnets region (I) and $\phi_{\mathrm{II}}$ in the air-gap region (II) are respectively the solution of the Poisson and Laplace equations. In Cartesian coordinates, they are given by:

$$
\nabla^{2} \phi_{I}=\nabla \cdot \vec{M}=\frac{\partial M_{x}}{\partial x}, \quad \nabla^{2} \phi_{I I}=0
$$

Where $\vec{M}$ is the magnetization of the magnets.

By considering the previous boundary value problem, the magnetization distribution is obtained by expanding it into a double Fourier series along $x$ - and $y$-directions (Fig. 3) as follows:

$$
\begin{aligned}
& M_{x}(x, y)=\sum_{n=1}^{\infty} \sum_{m=1}^{\infty} M_{x(n, m)} \cos \left(w_{n} x\right) \cos \left(w_{m} y\right) \\
& M_{z}(x, y)=\sum_{n=1}^{\infty} \sum_{m=1}^{\infty} M_{z(n, m)} \sin \left(w_{n} x\right) \cos \left(w_{m} y\right)
\end{aligned}
$$

With;

$$
\begin{gathered}
M_{x(n, m)}=\frac{16 B_{r}}{\mu_{0} n m \pi^{2}} \sin \left(w_{n} a\right) \sin \left(w_{m} b\right) \\
M_{z(n, m)}=\frac{16 B_{r}}{\mu_{0} n m \pi^{2}} \sin \left(w_{n} a\right) \sin \left(\frac{n \pi}{2}\right) \sin \left(w_{m} b\right)
\end{gathered}
$$

and

$$
w_{n}=\frac{n \pi}{\left(4 a+2 e_{g}\right)}, \quad w_{m}=\frac{m \pi}{2 l_{y}}
$$

Where $m, n$ are odd integers and $B_{r}$ is the remanent flux density.

Using the method of separation of variables, the general solutions of the magnetic scalar potential $\left(\phi_{\mathrm{I}}, \phi_{\mathrm{II}}\right)$, can be written as: 


$$
\begin{gathered}
\phi_{I}(x, y, z)=\mathfrak{R}\left[\sum_{n=1 m=1}^{\infty} \sum_{m=1}^{\infty}\left(A_{I} e^{k z}+B_{I} e^{-k z}-\frac{j M_{x(n, m)} w_{n}}{k^{2}}\right) \cos \left(w_{m} y\right) e^{j w_{n} x}\right] \\
\phi_{I I}(x, y, z)=\mathfrak{R}\left[\sum_{n=1}^{\infty} \sum_{m=1}^{\infty}\left(A_{I I} e^{k z}+B_{I I} e^{-k z}\right) \cos \left(w_{m} y\right) e^{j w_{n} x}\right]
\end{gathered}
$$

With; $\quad k=\sqrt{w_{n}^{2}+w_{m}^{2}}$

The problem in the conductive region (III) is solved using an H-formulation. The reference frame is fixed to the magnets region and the field source is static. So we can write:

$$
\nabla^{2} H_{I I I}=-\sigma \mu_{0} \nabla \times\left(V \times H_{I I I}\right)
$$

Where $\sigma$ is the conductivity and $V=v_{x}$ the velocity of the conducting plate.

In a Cartesian coordinate system, the development of (6) gives us three partial differential equations (PDEs):

$$
\begin{aligned}
& \frac{\partial^{2} H_{x}}{\partial x^{2}}+\frac{\partial^{2} H_{x}}{\partial y^{2}}+\frac{\partial^{2} H_{x}}{\partial z^{2}}=\sigma \mu_{0} v_{x} \frac{\partial H_{x}}{\partial x} \\
& \frac{\partial^{2} H_{y}}{\partial x^{2}}+\frac{\partial^{2} H_{y}}{\partial y^{2}}+\frac{\partial^{2} H_{y}}{\partial z^{2}}=\sigma \mu_{0} v_{x} \frac{\partial H_{y}}{\partial x} \\
& \frac{\partial^{2} H_{z}}{\partial x^{2}}+\frac{\partial^{2} H_{z}}{\partial y^{2}}+\frac{\partial^{2} H_{z}}{\partial z^{2}}=\sigma \mu_{0} v_{x} \frac{\partial H_{z}}{\partial x}
\end{aligned}
$$

Considering the boundary's conditions along $x$ - and $y$-directions, the magnetic field strength in the region III $\left(\mathrm{H}_{x}, \mathrm{H}_{\mathrm{y}}, \mathrm{H}_{\mathrm{z}}\right)$, is obtained using the method of separation of variables:

$$
\begin{aligned}
& H_{x}(x, y, z)=\mathfrak{R}\left[\sum_{n=1}^{\infty} \sum_{m=1}^{\infty}\left(A_{x I I I} e^{\alpha \cdot z}+B_{x I I I} e^{-\alpha \cdot z}\right) \cos \left(w_{m} y\right) e^{j w_{n} x}\right] \\
& H_{z}(x, y, z)=\mathfrak{R}\left[\sum_{n=1}^{\infty} \sum_{m=1}^{\infty}\left(A_{z I I I} e^{\alpha \cdot z}+B_{z I I I} e^{-\alpha \cdot z}\right) \cos \left(w_{m} y\right) e^{j w_{n} x}\right]
\end{aligned}
$$

From the expression ( $\operatorname{div} B=0$ ) we can find the expression of $H y$ given by:

$$
H_{y}(x, y, z)=-\int\left(\frac{\partial H_{x}}{\partial x}+\frac{\partial H_{z}}{\partial z}\right) d y
$$

Substituting (10) and (11), in (12), we obtain:

$$
H_{y}(x, y, z)=\mathfrak{R}\left[\sum_{n=1}^{\infty} \sum_{m=1}^{\infty} \frac{-1}{w_{m}}\left(j w_{n}\left(A_{x I I I} e^{\alpha \cdot z}+B_{x I I I} e^{-\alpha \cdot z}\right)+\alpha\left(A_{z I I I} e^{\alpha \cdot z}-B_{z I I I} e^{-\alpha \cdot z}\right)\right) \sin \left(w_{m} y\right) e^{j w_{n} x}\right]
$$

With;

$$
\alpha=\sqrt{w_{n}^{2}+w_{m}^{2}+j \sigma \mu_{0} v_{x} w_{n}}
$$


The analytical expressions of the complex coefficients, $A_{I}, B_{I}, A_{I I}, B_{I I}, A_{x I I I}, B_{x I I I}, A_{z I I I}$ and $B_{z I I I}$ are determined using the boundary's and interfaces conditions between the different regions given by:

Boundary conditions at $z=0$ and $z=z_{3}=h_{a}+e+h_{p}$ (Fig.2):

$$
\left\{\begin{array}{l}
\phi_{I}(x, y, 0)=0 \\
H_{x I I I}\left(x, y, z_{3}\right)=0 \\
\frac{\partial H_{z I I I}\left(x, y, z_{3}\right)}{\partial z}=0
\end{array}\right.
$$

Interfaces conditions between the different regions (I, II, II) (Fig.2):

At $z=z_{1}=h_{a}$ :

$$
\left\{\begin{array}{l}
\phi_{I}\left(x, y, z_{1}\right)=\phi_{I I}\left(x, y, z_{1}\right) \\
\frac{\partial \phi_{I}\left(x, y, z_{1}\right)}{\partial z}=\frac{\partial \phi_{I I}\left(x, y, z_{1}\right)}{\partial z}-M_{z}(x, y)
\end{array}\right.
$$

At $z=z_{2}=h_{a}+e:$

$$
\left\{\begin{array}{l}
\frac{\partial \phi_{I I}\left(x, y, z_{2}\right)}{\partial x}=-H_{x I I I}\left(x, y, z_{2}\right) \\
\frac{\partial \phi_{I I}\left(x, y, z_{2}\right)}{\partial y}=-H_{y I I I}\left(x, y, z_{2}\right) \\
\frac{\partial \phi_{I I}\left(x, y, z_{2}\right)}{\partial z}=-H_{z I I I}\left(x, y, z_{2}\right)
\end{array}\right.
$$

The analytical expressions of the unknown coefficients are given in the Appendix.

\subsection{Eddy currents expressions in conductive plate}

The eddy currents in an infinite conductive plate are computed using the Ampere law and is given by:

$$
\overrightarrow{J_{I I I}}=\overrightarrow{\operatorname{curl}}\left(\overrightarrow{H_{I I I}}\right)
$$

Substituting the expressions of the magnetic field strength (10), (11), (13) into (17), after calculation, the components of the induced current density in the conducting plate are given as follows:

$$
\begin{gathered}
J_{x}(x, y, z)=\mathfrak{R}\left[\sum_{n=1}^{\infty} \sum_{m=1}^{\infty}\left(\frac{w_{m}^{2}-\alpha}{w_{m}}\left(A_{z I I I} e^{\alpha \cdot z}+B_{z I I I} e^{-\alpha \cdot z}\right)-\frac{j \alpha w_{n}}{w_{m}}\left(A_{x I I I} e^{\alpha \cdot z}-B_{x I I I} e^{-\alpha \cdot z}\right)\right) \sin \left(w_{m} y\right) e^{j w_{n} x}\right] \\
J_{y}(x, y, z)=\mathfrak{R}\left[\sum_{n=1}^{\infty} \sum_{m=1}^{\infty}\left(j w_{n}\left(A_{z I I I} e^{\alpha \cdot z}+B_{z I I I} e^{-\alpha \cdot z}\right)-\alpha\left(A_{x I I I} e^{\alpha \cdot z}-B_{x I I I} e^{-\alpha \cdot z}\right)\right) \cos \left(w_{m} y\right) e^{j w_{n} x}\right] \\
J_{z}(x, y, z)=0
\end{gathered}
$$


In the case of finite dimensions, the normal components of eddy currents at lateral faces of the conducting plate are vanish. To satisfy these boundary's conditions with the concept of images method (Messadi \& al., 2016; Pluk \& al., 2014; Zhang \& al., 2014), an infinite multiplication of images can be introduced outside the plate in $y$-direction. Thus, the eddy current density produced in a point of the plate with finite dimensions is the superposition of the source eddy current density calculated in the case of an infinite width plate and the eddy current densities of the images (Fig.4).

In our model the boundary's conditions on the lateral faces of the conducting plate are taken into account by exploiting the fictitious poles imposed by the fictitious anti-periodic condition on the $y$-direction. So to apply the image's eddy current densities it is enough to impose $l y=b$ (Fig. 3).

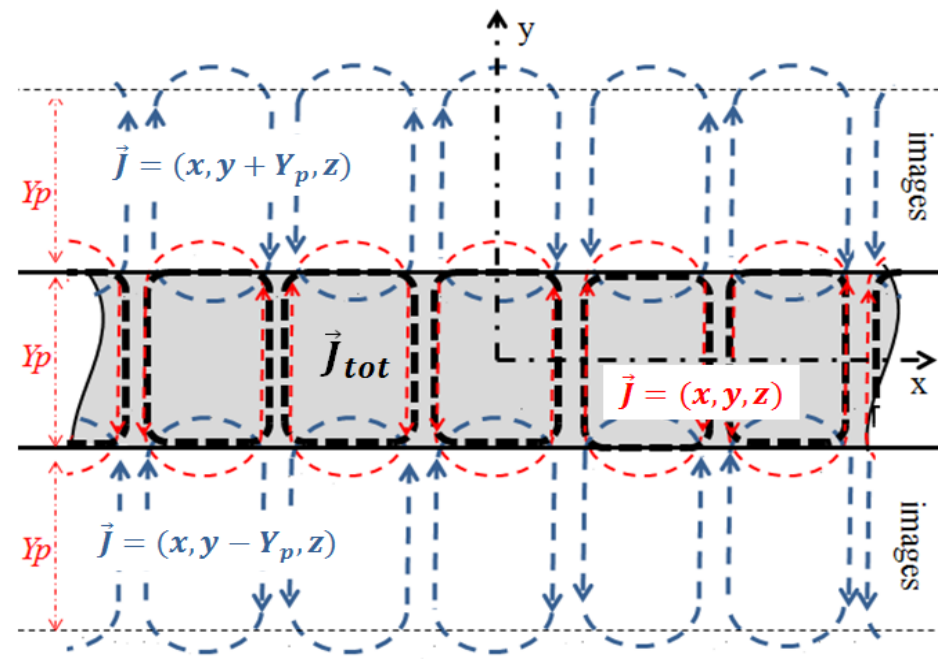

Fig. 4. The current densities involved for one layer of images.

The induced current density distribution, for one layer of images, is shown in the fig.4, where $\vec{J}_{\text {tot }}$ is the real distribution of the eddy current density (conducting plate of finite dimensions with transverse edge effect), resulting from the current density calculated for an infinite dimensions conducting plate $\vec{J}(x, y, z)$, and that of images $\vec{J}\left(x, y \pm Y_{p}, z\right)$.

\subsection{Heating power}

The heating power, due to the induced currents is computed by the integration of the power density in the volume of the conducting plate as follows:

$$
P(t)=\int_{-X_{p} / 2}^{X_{p} / 2} \int_{h_{a}+e}^{h_{p}} \int_{-Y_{p} / 2}^{Y_{p} / 2} 2 \cdot\left(\frac{J_{x}^{2}+J_{y}^{2}}{\sigma}\right) d x d z d y
$$

Where $X_{p}, Y_{p}$ and $2 h_{p}$ are respectively the length, the width and the thickness of the conducting plate, Fig.1.

\section{Results}

In this section, we use the proposed 3D analytical model to analyze the performances of the studied induction heater. In order to show the accuracy of the 3-D analytical model, the results are compared with those obtained from 3D FEM implemented in COMSOL-Multiphysics software and experimental measurements performed on the test rig shown in Fig.5. 
To perform the 3D finite element computations with COMSOL software, a 3D mesh of 841263 tetrahedral elements leading to solve a global algebraic system having 5360005 degrees of freedom is used. Obviously, the 3D model take into account all the edge effects.

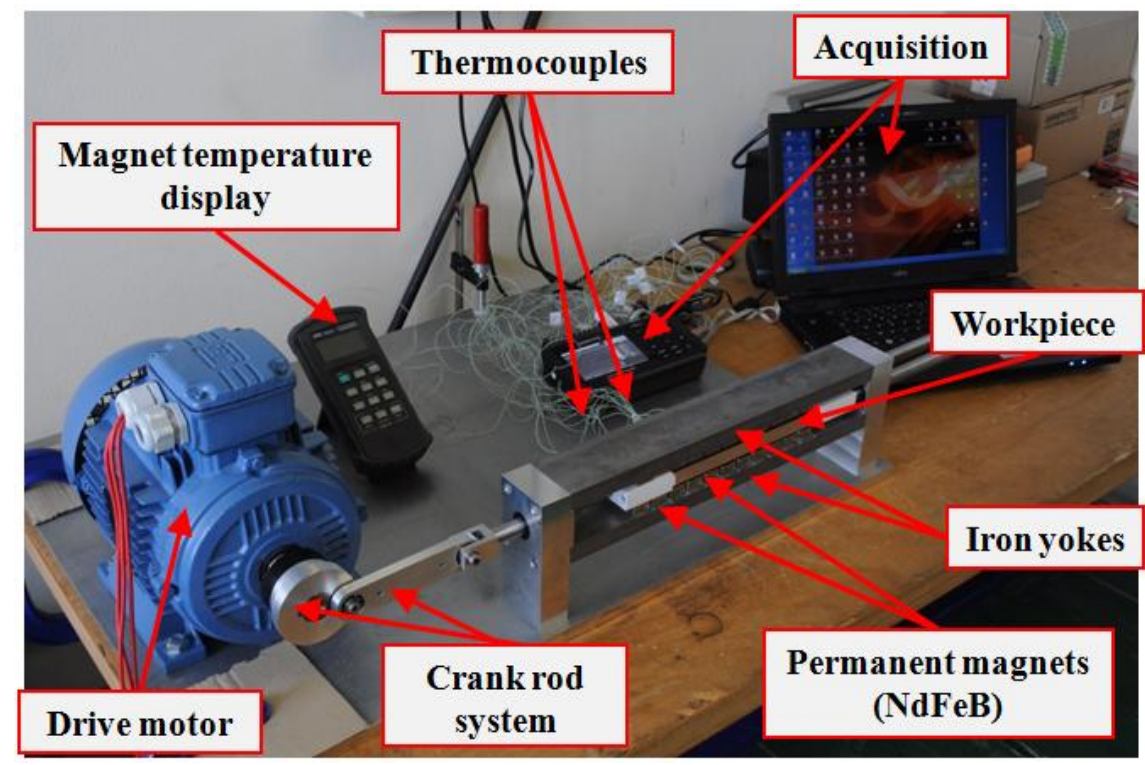

Fig. 5. Induction heater prototype.

The oscillatory linear motion of the workpiece is obtained by transformation of rotary motion using a crank-rod system (Fig.5). Fig.6 gives the linear velocity evolution versus time for a peak value Vmax $=0.5 \mathrm{~m} / \mathrm{s}$. We can observe that the linear velocity presents a sinusoidal waveform.

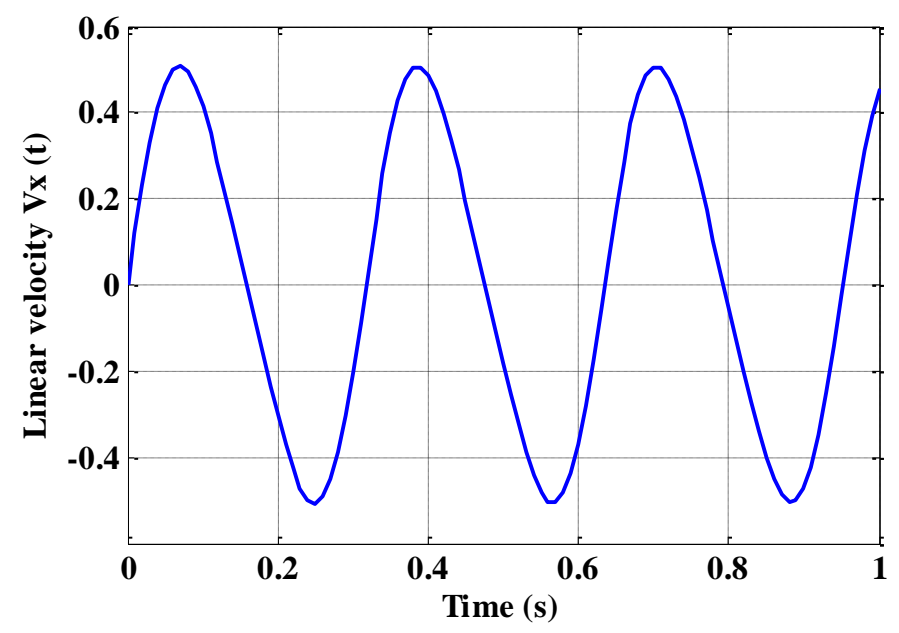

Fig. 6. Linear velocity evolution versus time.

The normal magnetic flux densities distribution $(B z)$ in the middle of the air-gap at $z=h_{a}+e / 2$, along the $\mathrm{x}$-direction and $\mathrm{y}$ direction, are presented in Fig.7 and Fig.8 respectively. These results are obtained at rest.

A very good agreement is noticed between the analytical results and those obtained with the finite element simulation and the experimental measurements. 


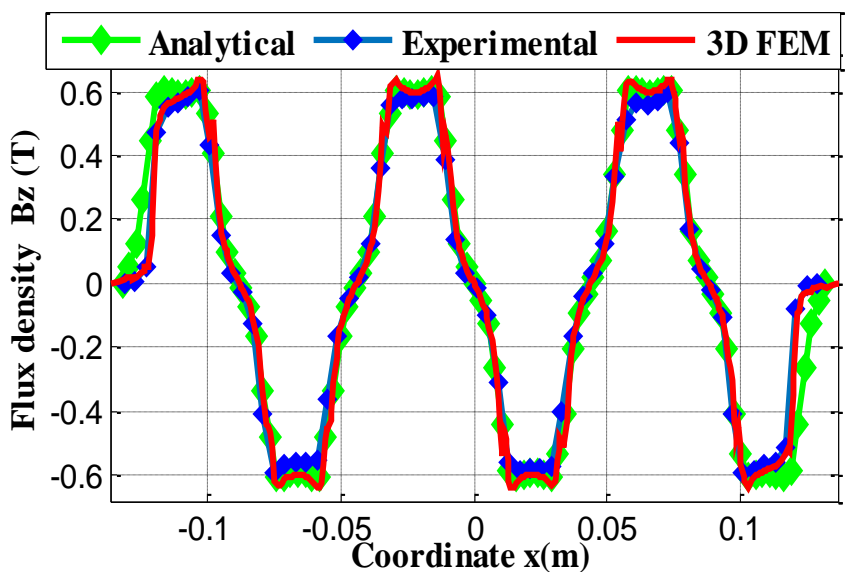

Fig. 7. Normal flux density $(B z)$ along the $\mathrm{x}$-direction in the middle of the air gap.

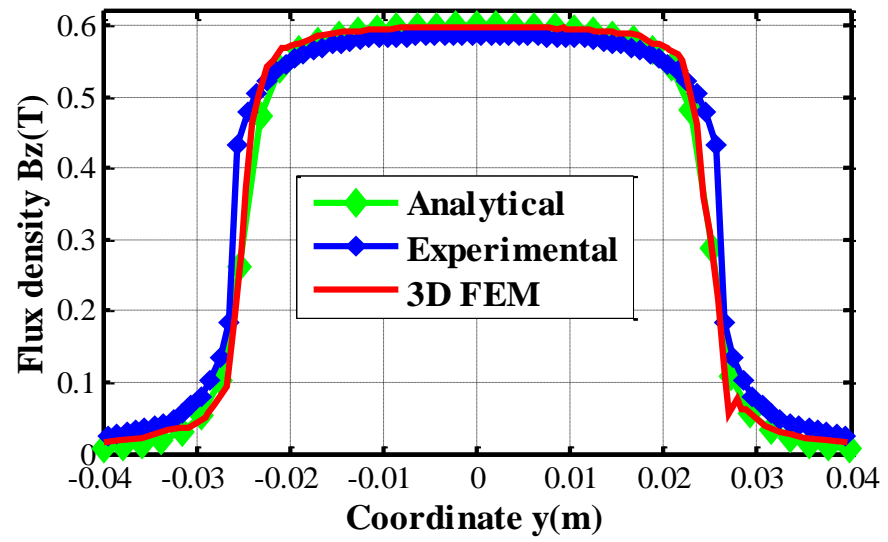

Fig. 8. Normal flux density $(B z)$ along the $y$-direction in the middle of the air gap.

Fig.9 and Fig.10 show respectively the distribution of the flux density component $B_{z}$ along the x-direction and y-direction, on workpiece surface at $\mathrm{z}=\mathrm{z}_{2}\left(z_{2}=h_{a}+e\right)$ and in the middle of the workpiece at $\mathrm{z}=\mathrm{z}_{3}\left(z_{3}=h_{a}+e+h_{p}\right)$ for a velocity peak of $0.5 \mathrm{~m} / \mathrm{s}$. We can observe that the flux density is little variable across the thickness of the workpiece.

The flux density waveforms predicted by the $3 \mathrm{D}$ developed model are in a good agreement compared to the finite element simulation.

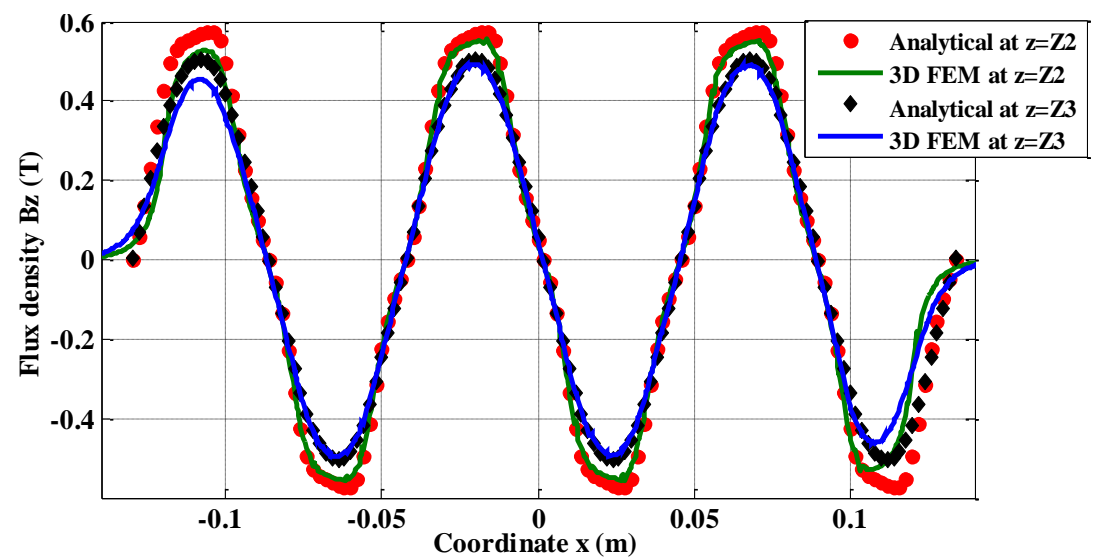

Fig. 9. Normal flux density $(B z)$ along the $\mathrm{x}$-direction on workpiece surface at $\mathrm{z}=\mathrm{z}_{2}$ and in the middle of the workpiece at $\mathrm{z}=\mathrm{z}_{3}$. 


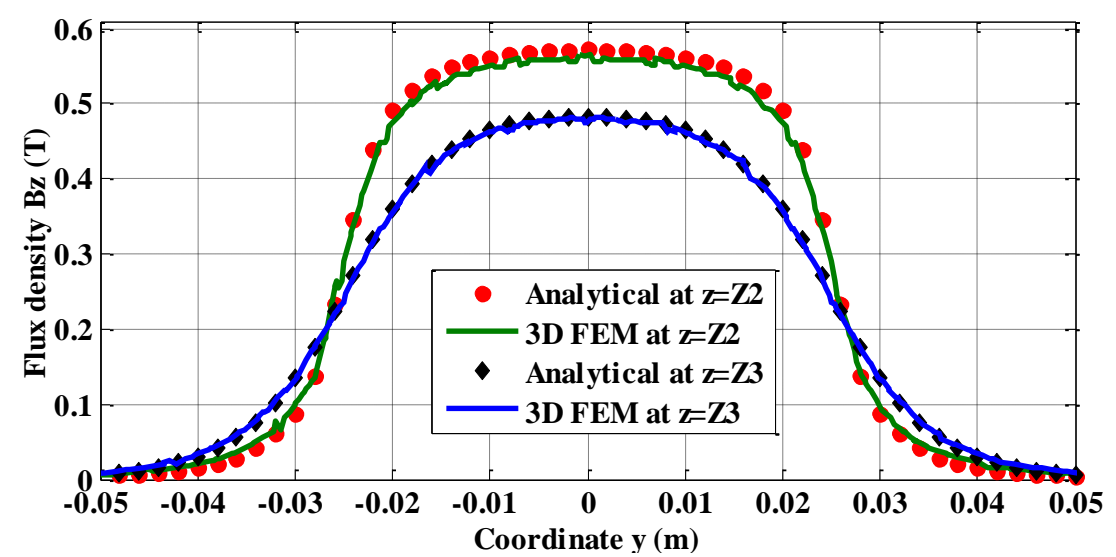

Fig. 10. Normal flux density $(B z)$ along the $\mathrm{y}$-direction on workpiece surface at $\mathrm{z}=\mathrm{z}_{2}$ and in the middle of the workpiece at $\mathrm{z}=\mathrm{z}_{3}$.

To show the effect of the reaction field due to induced currents, we compare the normal flux density distribution $(B z)$ along the $\mathrm{x}$-direction obtained at rest and with a velocity peak of $2 \mathrm{~m} / \mathrm{s}$ (linear speed with sinusoidal variation) (Fig.11). It can be seen that the reaction field due to the induced currents in the conducting plate tends to shift and distort the flux density.

The analytical results show a good agreement with those calculated by the finite element method.

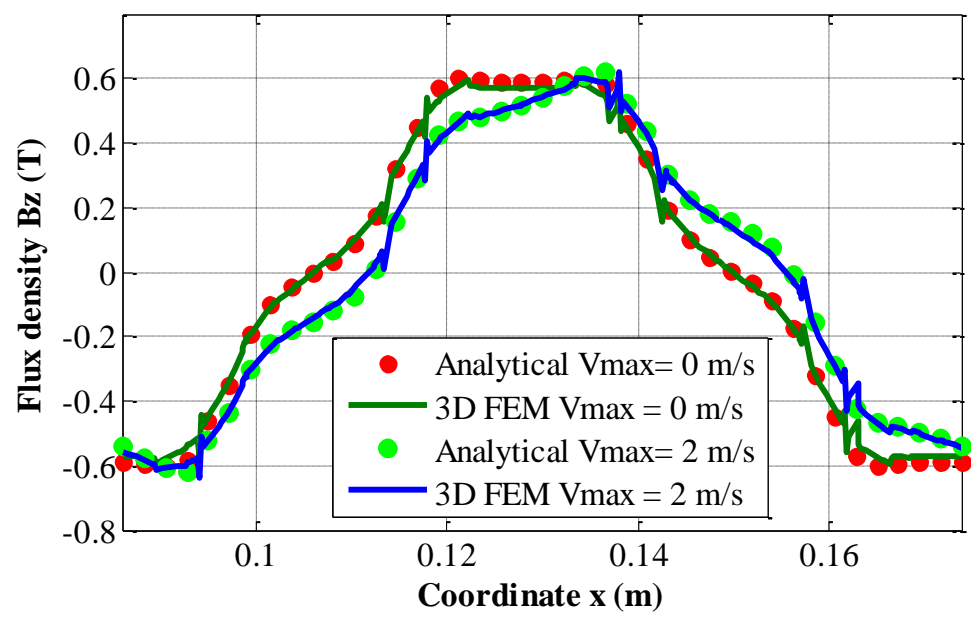

Fig. 11. Normal flux density (Bz) along the x-direction in the middle of the air gap under two pole, for $V \max =0 \mathrm{~m} / \mathrm{s}$ and $\mathrm{Vmax}=2 \mathrm{~m} / \mathrm{s}$.

Fig.12 and Fig.13 show respectively the distribution of the induced current density components $J x$ and $J y$, along the $\mathrm{x}$ direction (a) and y-direction (b), calculated by the analytical model, with and without the application of the image's method.

These eddy currents densities are calculated in the middle of the aluminum conducting plat $\left(\sigma=33.10^{6} \mathrm{Sm}^{-1}\right)$ at $\left(z=h_{a}+\right.$ $e+h_{p}$ ), for a velocity $\mathrm{V} \max =0.5 \mathrm{~m} / \mathrm{s}$.

It is noted that for an infinite width conducting plate, the obtained results underestimate the induced current component $J x$ and overestimate the component $J y$, compared to those calculated by the finite element method.

However, after applying the image's method to take into account the real dimensions of the conducting plate, along the ydirection, the proposed model with image's gives very similar results compared to the 3D finite element simulations. 


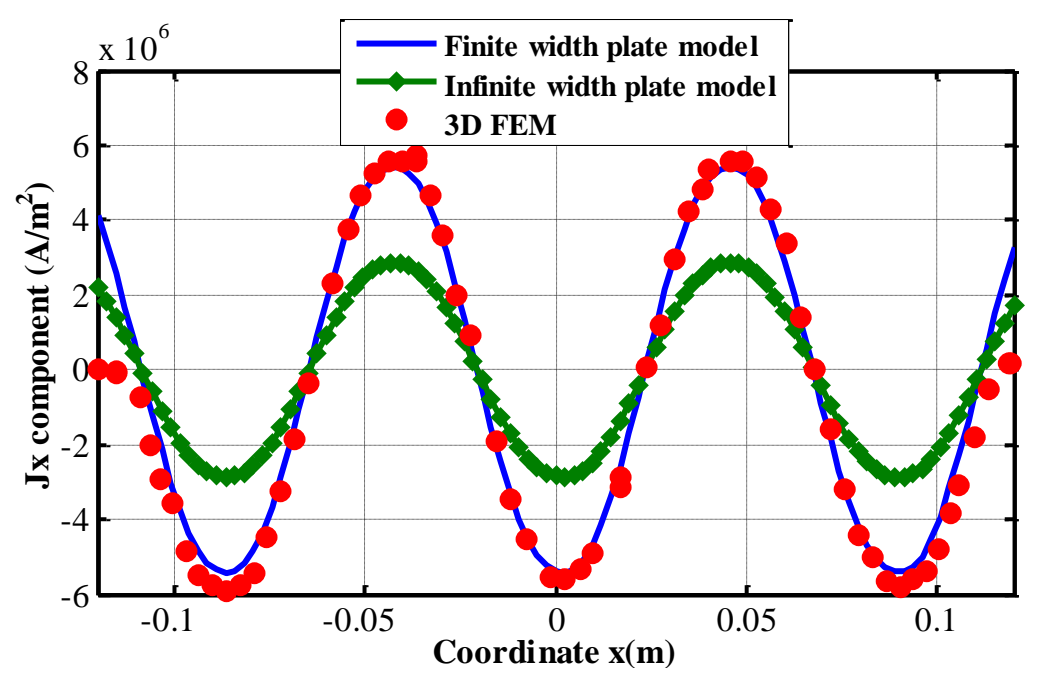

(a)

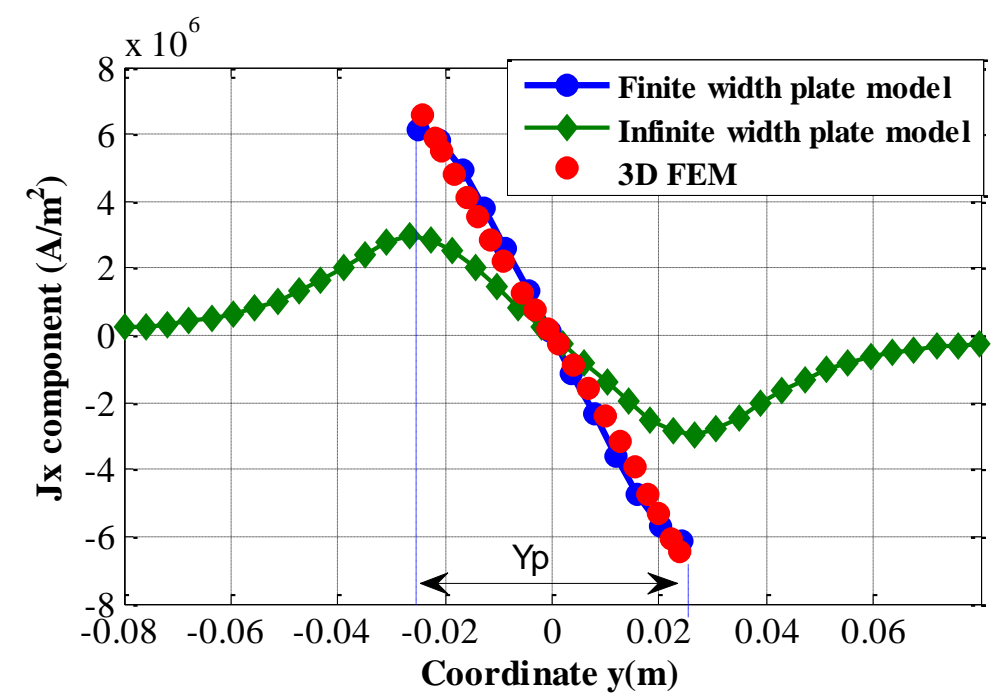

(b)

Fig. 12. Induced current density distribution $(\mathrm{Jx})$ in the middle of the conducting plate for Vmax=0.5 $\mathrm{m} / \mathrm{s}$. $(a)$ along the $\mathrm{x}$-direction. $(\mathrm{b})$ along the $\mathrm{y}$-direction.

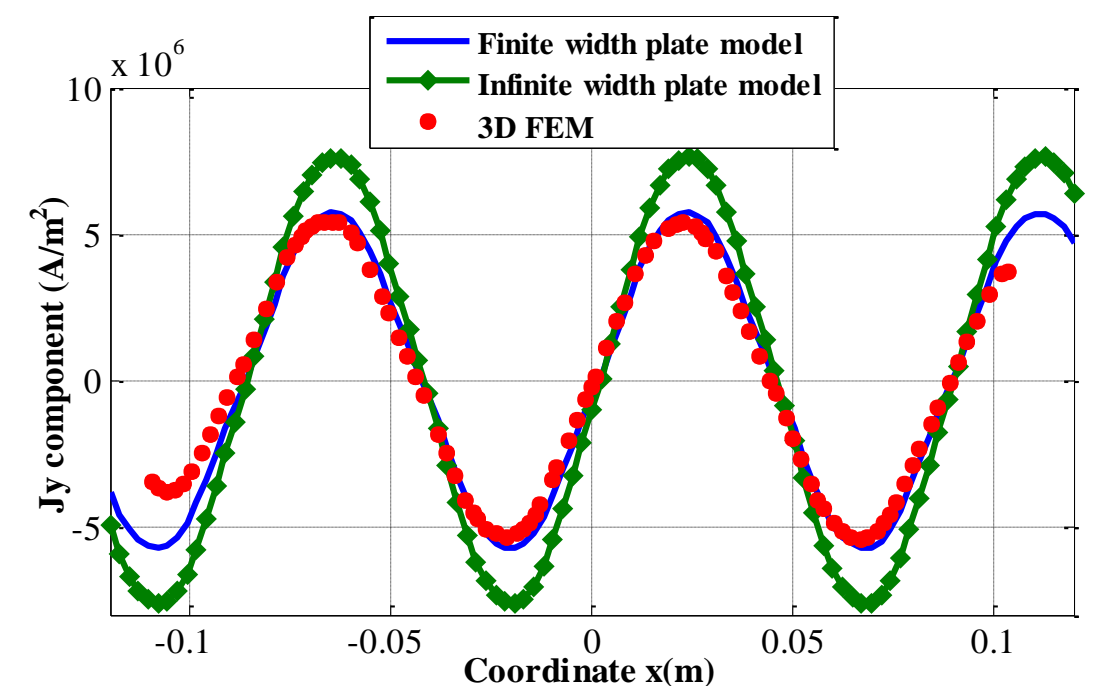

(a) 


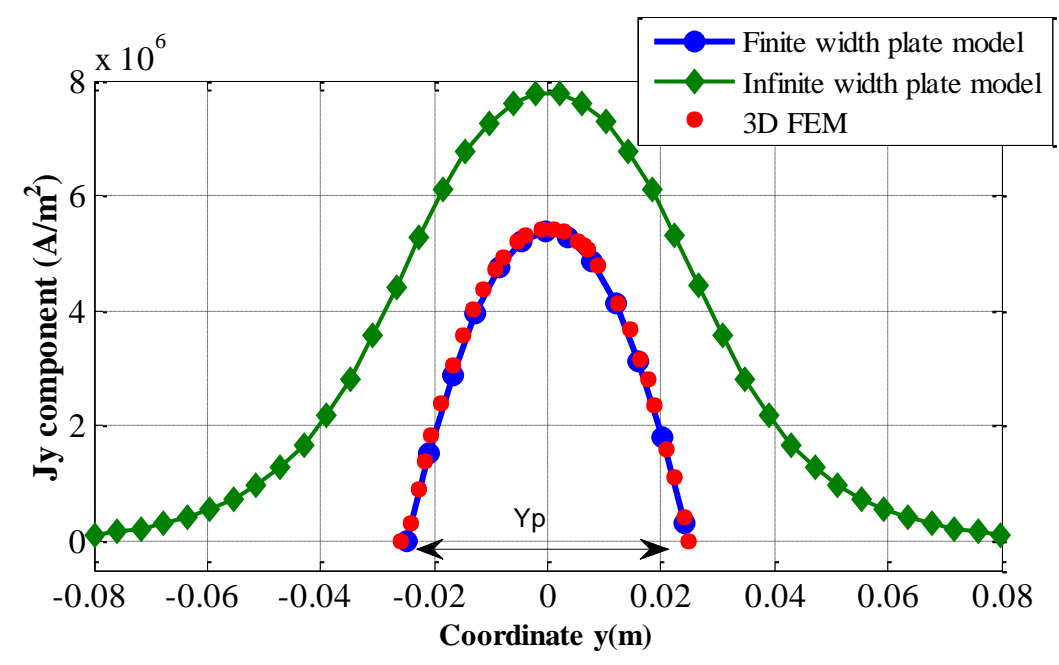

(b)

Fig. 13. Induced current density distribution $(\mathrm{Jy})$ in the middle of the conducting plate for Vmax $=0.5 \mathrm{~m} / \mathrm{s}$. $(a)$ along the $\mathrm{x}$-direction. $(b)$ along the $\mathrm{y}$-direction.

Fig.14(a) and Fig.14(b) show respectively the current density distributions $J_{x}$ and $J_{y}$ in $(x, y)$ plan at the middle of the workpiece obtained with the analytical model (finite width workpiece) for a velocity $\mathrm{Vmax}=0.5 \mathrm{~m} / \mathrm{s}$.

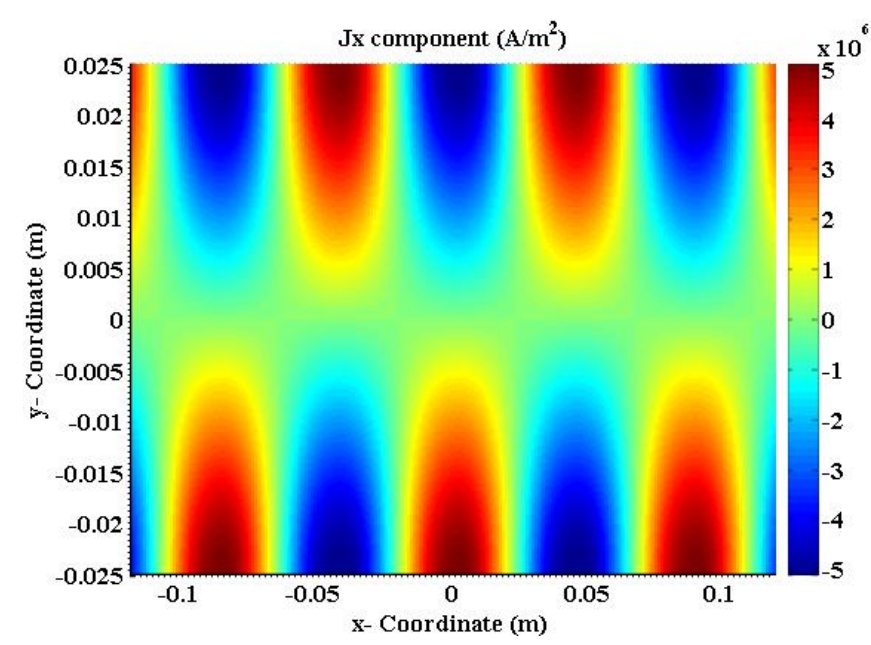

(a)

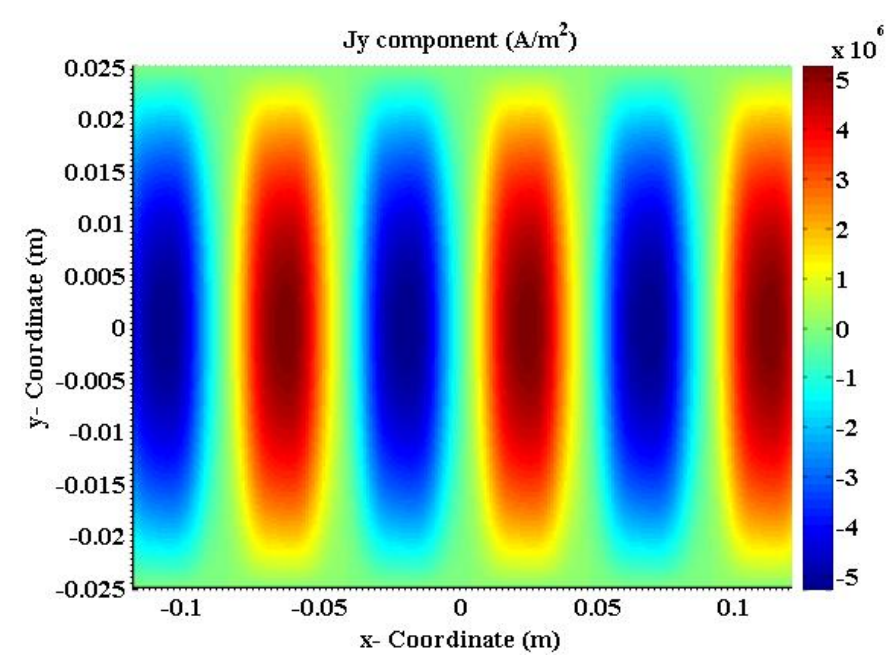

(b)

Fig. 14. Induced current density distribution in $x y$ plane at the middle of the workpiece for $\operatorname{Vmax}=0.5 \mathrm{~m} / \mathrm{s}$ : (a) component $J_{x}$, (b) component $J_{y}(\mathrm{Vmax}=0.5 \mathrm{~m} / \mathrm{s})$.

The average induced heating power in the aluminum conducting plate as a function of peak velocity values (Vmax) is shown in Fig. 15.

The results obtained before and after the use of the image's method, along the y-direction, are compared with those obtained from the 3-D finite element simulations and experimental measurements. Again, it can be seen that for an infinite conducting plate (neglecting the finite length effects) the obtained results over estimate the heating power (error about $45 \%$ compared to the measured value at $1.2 \mathrm{~m} / \mathrm{s}$ ). After the use of the image's method, our model gives very close results compared to the 3D finite element simulations and to the measurements. 


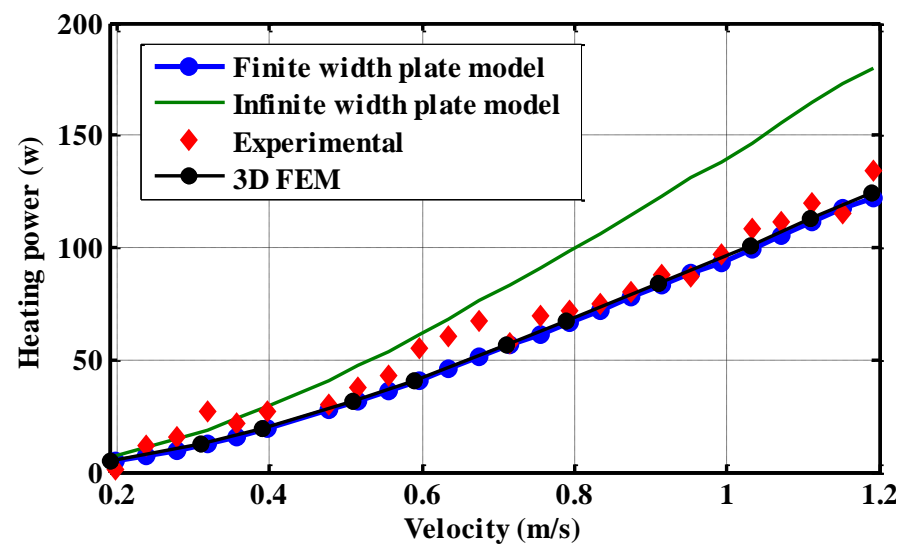

Fig. 15. Heating power in the conducting plate versus peak velocity values (Vmax).

Fig.16 gives the relative error on the heating power calculation between the analytical model (finite and infinite width workpiece) and the 3-D FE model versus peak value of linear velocity.

It can be seen that for an infinite width conducting plate (neglecting the finite length effects) the error varies between $40 \%$ and $45 \%$ for peak velocity values ranging from $0.2 \mathrm{~m} / \mathrm{s}$ to $1.2 \mathrm{~m} / \mathrm{s}$. However, after applying the image's method (finite width conducting plate) the error doesn't exceed $2 \%$ at a speed of $1.2 \mathrm{~m} / \mathrm{s}$.

The proposed model presents an important reduction in computation time. Indeed, for a given velocity, the calculation time for the 3D finite element method is about 196s, while that of the analytic model is 0.39 s. So the proposed model can be used as an effective tool in a design optimization procedure of the induction heater. We note that for all analytical calculations we have considered a same number of harmonics in the $\mathrm{x}$ and $\mathrm{y}$ directions, $\mathrm{n}=25$ and $\mathrm{m}=25$.

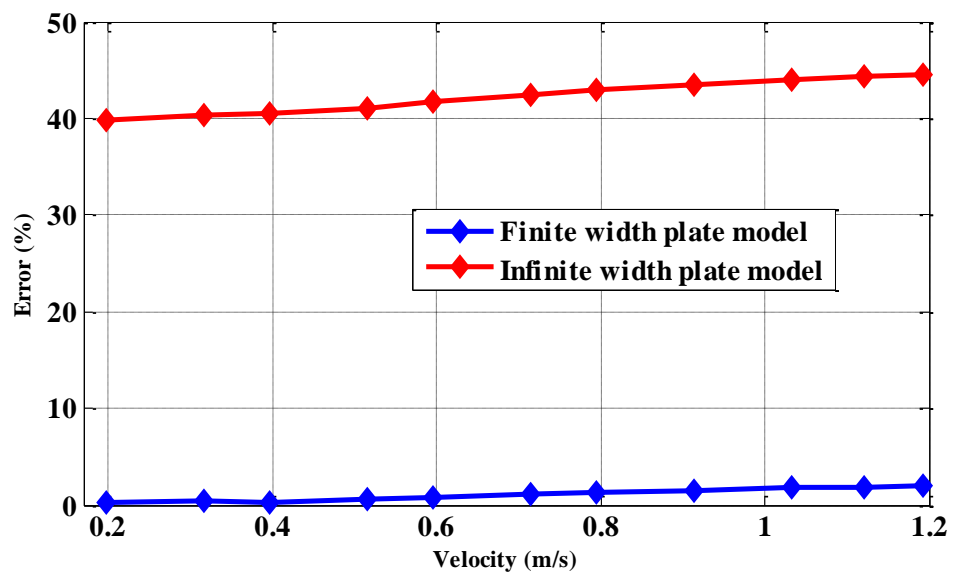

Fig. 16. Error on the heating power calculation between the analytical model and the 3-D FE model versus peak velocity values (Vmax).

\section{Conclusion}

In this paper, a 3-D electromagnetic model based on sub-domain's method and the image's theory is developed for calculating the eddy currents and the heating power, dissipated by Joule effect, in a conductive plate with consideration of finite length effects and the eddy-current reaction field. 
The results show the accuracy of the developed model to properly account for the transverse edge effects. The results are in good agreement with those obtained by 3D FEM and experiments. The calculation times are considerably reduced using the proposed method.

\section{APPENDIX}

The analytical expressions of the complex coefficients, $A_{I}, B_{I}, A_{I I}, B_{I I}, A_{x I I I}, B_{x I I I}, A_{z I I I}$ and $B_{z I I I}$ are given by:

$A_{I I}=\frac{C 1}{C 2}, \quad B_{I I}=-A_{I I}\left(\frac{S 1}{S 2}\right) e^{2 k z_{2}}$

$A_{I}=A_{I I} \frac{e^{k z_{1}}}{2 \sinh \left(k z_{1}\right)}+B_{I I} \frac{e^{-k z_{1}}}{2 \sinh \left(k z_{1}\right)}+\frac{j M_{x(n, m)}}{k^{2}} \frac{1}{2 \sinh \left(k z_{1}\right)}-\frac{j M_{x(n, m)} w_{n}}{k^{2}} \frac{e^{-k z_{1}}}{2 \sinh \left(k z_{1}\right)}, \quad B_{I}=-A_{I}+\frac{j M_{x(n, m)} w_{n}}{k^{2}}$

$A_{x I I I}=-A_{I I} \frac{j w_{n} e^{k z_{2}}}{e^{\alpha z_{2}}-e^{-\alpha z_{2}} e^{2 \alpha z_{3}}}-B_{I I} \frac{j w_{n} e^{-k z_{2}}}{e^{\alpha z_{2}}-e^{-\alpha z_{2}} e^{2 \alpha z_{3}}}, \quad B_{x I I I}=-A_{x I I I} e^{2 \alpha z_{3}}$

$A_{z I I I}=-A_{I I} \frac{k e^{k z_{2}}}{e^{\alpha z_{2}}+e^{-\alpha z_{2}} e^{2 \alpha z_{3}}}+B_{I I} \frac{k e^{-k z_{2}}}{e^{\alpha z_{2}}+e^{-\alpha z_{2}} e^{2 \alpha z_{3}}}, \quad B_{Z I I I}=A_{z I I I} e^{2 \alpha z_{3}}$

With:

$$
\begin{aligned}
& S 1=\frac{-w_{m}^{2}-w_{n}^{2}}{w_{m}}+\frac{\alpha k}{w_{m}}\left(\frac{\mathrm{e}^{\alpha z_{2}}-\mathrm{e}^{-\alpha z_{2}} \mathrm{e}^{2 \alpha z_{3}}}{\mathrm{e}^{\alpha z_{2}}+\mathrm{e}^{-\alpha z_{2}} \mathrm{e}^{2 \alpha z_{3}}}\right) \\
& S 2=\frac{-w_{m}^{2}-w_{n}^{2}}{w_{m}}-\frac{\alpha k}{w_{m}}\left(\frac{\mathrm{e}^{\alpha z_{2}}-\mathrm{e}^{-\alpha z_{2}} \mathrm{e}^{2 \alpha z_{3}}}{\mathrm{e}^{\alpha z_{2}}+\mathrm{e}^{-\alpha z_{2}} \mathrm{e}^{2 \alpha z_{3}}}\right) \\
& C 1=\frac{j M_{x(n, m) w_{n}}}{k^{2}}\left(\frac{\cosh \left(k z_{1}\right)}{\sinh \left(k z_{1}\right)}\left(e^{-k z_{1}}-1\right)+e^{-k z_{1}}\right)-\frac{j M_{z(n, m)}}{k} \\
& C 2=\left(\frac{\cosh \left(k z_{1}\right)}{\sinh \left(k z_{1}\right)}-1\right) e^{k z_{1}}-\left(\frac{S 1}{s 2}\right)\left(\frac{\cosh \left(k z_{1}\right)}{\sinh \left(k z_{1}\right)}+1\right) e^{k\left(2 z_{2}-z_{1}\right)}
\end{aligned}
$$

\section{REFERENCES}

V. Rudnev, D. Loveless, R. L. Cook, and M. Black, "Handbook of Induction Heating”, New York, NY, USA: Marcel Dekker, 2003.

N. Magnusson, M. Runde, "Efficiency Analysis of a High Temperature Superconducting Induction Heater," IEEE Trans. appl. supercond., vol. 13, no. 2, pp.1616-1619, Jun.2003.

T. Lubin, D. Netter, J. Leveque, and A. Rezzoug, "Induction heating of aluminum billet subjected to a strong rotating magnetic field produced by superconducting windings," IEEE Trans. on Magn., vol. 45, no 5, pp. 2118-2127, May 2009.

M. Messadi, L. Hadjout, Y. Ouazir, T. Lubin, S. Mezani, A. Rezzoug, and N. Takorabet, "Eddy current computation in translational motion conductive plate of an induction heater with consideration of finite length extremity effects," IEEE Trans.Magn,, vol. 52, Issue: 3, 1-4, March 2016.

H.Bensaidane, T.Lubin, S.Mezani, Y.Ouazir, and A.Rezzoug, "A New topology for induction heating system with PM excitation: electromagnetic model and experimental Validations," IEEE Trans. Magn., vol. 51, no. 10, October 2015.

M. Fabbri, M. Forzan, S. Lupi, A. Morandi, and P. L. Ribani, "Experimental and numerical analysis of DC induction heating of aluminum billets," IEEE Trans. Magn., vol. 45, no. 1, pp. 192-200, Jan. 2009.

F. Mach, P. Karban, and I. Doležel, "Induction heating of cylindrical nonmagnetic ingots by rotation in static magnetic field generated by permanent magnets," $J$. Comput. Appl. Math., vol. 236, no. 18, pp. 4732-4744, Dec. 2012.

M. Fabbri, A. Morandi, F. Negrini, "Temperature distribution in aluminum billets heated by rotation in static magnetic field produced by superconducting magnets," COMPEL., vol. 24, no. 1, pp.281-290, 2005. 
F. Mach, P. Karban, I. Dolezel, P. Sima, and Z. Jelinek, "Model of induction heating of rotating non-magnetic billets and its experimental verification," IEEE Trans. Magn., vol. 50, no. 2, Feb. 2014.

A.H.Selçuk , H. Kürüm, "Investigation of end effects in linear induction motors by using the finite element method," IEEE TransMagn, vol. 44, No. 7,pp17911795, July 2008.

O. Bíró, G. Koszka and K. Preis, "Fast time-domain finite element analysis of 3-D nonlinear time periodic eddy current problems with T, Ф-Ф formulation," IEEE Trans.Magn, vol 45, no. 5, pp.1170-1173, May 2011.

P. Karban, F. Mach, I. Dolezel, and J. Barglik, "Higher-order finite element modeling of rotational induction heating of nonferromagnetic cylindrical billets," COMPEL, Int. J. Comput. Math. Elect. Electron. Eng., vol. 30, no. 5, pp. 1517-1527, 2011.

T. Lubin, and A. Rezzoug, "3-D analytical model for axial-flux eddy-current couplings and brakes under steady-state conditions". IEEE Trans. Magn, volume 51, $\mathrm{N}^{\circ} 10,8203712,12$ pages, Octobre 2015.

S.Paul, J.Wright, and J.Z.Bird, “3-D steady-state eddy current damping and stiffness for a finite thickenss conductive plate,” IEEE Trans.Magn., vol 50,no.11,pp 6301404, November 2014.

Jun. H. Zhang and al,"Modeling and analysis of a novel planar eddy current damper,” Journal of Applied Physics 115, $17 E 709$ „January 2014.

K. J. W. Pluk, T. A. van Beek, J. W. Jansen, and E. A. Lomonova, "Modeling and Measurements on a Finite Rectangular Conducting Plate in an Eddy Current Damper," IEEE Trans. Industrial Electronics, vol 61, no.8, pp4061-4072, August 2014.

Jun.H. Zhang and al, "Modeling and analysis of a novel planar eddy current damper," Journal of Applied Physics 115, 17E709,January 2014.

M. Boubezari, Y. Ouazir et H. Bensaidane, “2D Semi-analytical Analysis of Aluminum Billets heated by Rotating Permanent Magnets Inductor', IEEE, 16th biennial Conference on the Computation of Electromagnetic Fields CEFC 2014, 25th - $28^{\text {th }}$ May 2014, Annecy, France. 